\title{
Case Study: Unlocking resemblance between tuberculosis and cancer
}

\section{Corresponding author:}

Dr. Sunita Kumari Yadav, Zoology Department, Daulat Ram College, Delhi University, New Delhi 110007, India, phone: +919891830020, e-mail: sunita33347@gmail.com, sunitayadav@dr.du.ac.in
Medical Research Journal 2020; Volume 5, Number 4, 286-289 DOI: 10.5603/MRJ.a2020.0042 Copyright (C) 2020 Via Medica ISSN 2451-2591

\begin{abstract}
Tuberculosis (TB) has often been found to mimic malignancy in clinical and radiological features, and therefore it is well known as a diagnostic chameleon. Although the differences between pulmonary TB and lung cancer have been highlighted by several reports, TB often gets misdiagnosed as lung cancer and vice versa. It is therefore vital to discern the imaging results specific to pulmonary TB and its diverse forms to distinguish it from lung cancer. In the present study, a 72-year old female (HIV-negative, non-smoker), complaining of persistent cough, low chest pain, pain around kidneys and significant weight loss up to $8 \mathrm{~kg}$ in 4 months, was advised to undergo X-ray examination along with CT scan. The CT scan results showed manifestation of ill-defined lesions along the right main bronchus with partial atelectasis and multiple scattered centrilobular nodules in the right upper lobe. There was also a well-defined lesion at the left adrenal gland. Also, several sub-centimetre mediastinal nodes were found. The preliminary assessment by the doctor indicated lung cancer, and therefore PET scan was advised for further confirmation, also to detect any metastasis of malignancy if lung cancer is confirmed. PET scan results ruled out lung cancer; afterwards, the TB test (AFB smear) was recommended by the doctor. The final reports of the test results confirmed tuberculosis. However, this is not the only case where tuberculosis has been found to mimic lung cancer; there have been several case studies reporting misdiagnosis of TB. A correct diagnosis could only be achieved through high-imaging techniques or invasive examination. The present case expanded the clinical knowledge in the diagnosis of TB and also invigorated clinicians to consider pulmonary TB in the differential diagnosis and treatment. The study also accentuates the importance of ${ }^{18}$ FFDG-PET to differentiate TB from lung cancer.
\end{abstract}

Key words: tuberculosis, lung cancer, AFB test, PET scan, lesion

Med Res J 2020; 5 (4): 286-289

\section{Introduction}

Tuberculosis is an infectious disease caused by Mycobacterium tuberculosis that mostly affects the lungs. According to the World Health Organization (WHO), in 2018, an estimated 10 million people were diagnosed with tuberculosis (TB) worldwide, and 1.5 million of them died from it (including 0.25 million people with HIV). India is considered a highly prevalent country for tuberculosis, with the highest TB burden (2.15 million TB cases) worldwide in 2018 (WHO, 2018 report) [1]. Globally, TB is one of the top 10 causes of death, and a leading cause from a single infectious agent (above HIV/AIDS). The diagnosis of TB is done by AFB smear testing for causative bacteria.

TB greatly mimics malignancy as clinical symptoms and radiographic characteristics of both the diseases are quite similar [2]. Since the treatment of TB and can- cer is entirely different, it is critical to report more case studies to find out the distinctions between neoplasm and TB. In the present report, we describe the case of a 72-year-old woman with pulmonary TB who was on the verge of being misdiagnosed with lung cancer.

\section{Case report}

The patient had reported persistent cough, chest pain (around diaphragm), weakness and unintended severe weight loss up to $8 \mathrm{~kg}$ in 4 months. The CT scan of her chest and abdomen showed multiple ill-defined nodules in the pulmonary region, lymph nodes and one nodule on the adrenal gland. Although the patient did not have any symptoms of fever, dyspnoea or haemoptysis, she had a medical history of hypertension and hypercholesterolemia. The patient was a non-smoker 
and non-drinker with no family history of lung cancer or any other type of cancer.

\section{Preliminary investigations}

The routine laboratory test showed borderline blood sugar while fasting (107 mg/dL; normal range: 74-99 mg/dL), borderline cholesterol (202 mg/dL; normal range: < 200), high LDL cholesterol (144 mg/dL; normal range: $<100 \mathrm{mg} / \mathrm{dL}$ ), high non-HDL cholesterol (142 mg/dL; normal range: < $130 \mathrm{mg} / \mathrm{dL}$ ), high phosphorous (5.24 mg/dL; normal range: $2.5-4.5 \mathrm{mg} / \mathrm{dL}$ ), marginally high urea $(46.0 \mathrm{mg} / \mathrm{dL}$; normal range: 17.0-43.0 $\mathrm{mg} / \mathrm{dL}$ ) and low chloride (96.3; normal range: 101-109 $\mathrm{mmol} / \mathrm{L})$. A low eosinophil count

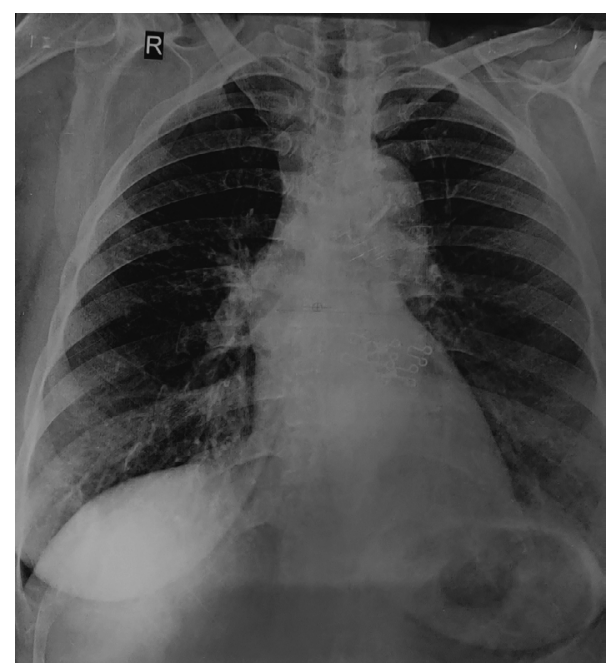

Figure 1. Chest X-ray showing infiltrates in the lungs primarily in the upper lung fields
(0.7\%; normal range: $1-6 \%$ ) and about two times higher ESR (normal range: $\leq 20$ ) was also detected in the haematology test. An abnormal increase in 25-hydroxy vitamin D (124.10 ng/mL; normal range: $30-100 \mathrm{ng} / \mathrm{mL}$ ) was also reported (the patient had been taking Calcirol 60,000 IU). The chest X-ray revealed the presence of several nodules primarily in the upper lung fields (Fig. 1).

The CT chest showed evidence of ill-defined soft tissue density lesion, measuring $4.6 \times 1.3 \mathrm{~cm}$, along the right main bronchus extending along and causing abrupt cut-off of right anterior segmental bronchiole with mildly associated atelectasis (Fig. 2). Also, there was evidence of scattered centrilobular infiltrates in the anterior as well as posterior segments of the right upper lobe. A tiny soft tissue density nodule and a tiny calcified nodule were also observed along the left oblique fissure and in the lateral basal segment of the left lower lobe, respectively. Although no cyst formation was seen, a sub centimetre mediastinal node was noted. The liver, gall bladder, pancreas, kidneys and spleen, however, were found to be normal; the left adrenal gland showed well-delineated iso to hypodense lesion with smooth margins. In the absence of diagnostic confirmation and suspicion of malignancy after a CT scan, the patient was advised to undergo positron emission tomography (PET).

PET indicated a few mild FDG avid right supraclavicular lymph nodes of the largest size $4 \mathrm{~mm}$ (SUV Max: 5). In the thorax region, multiple enlarged lymph nodes with lower attenuation showed evidence of internal calcification. The largest lymph node was $1.5 \mathrm{~cm}$ (SUV Max: 11) in the subcranial region (Fig. 3).

In the lung region, an FDG avid (SUV Max: 11), poorly marginated, low attenuation FDG avid consolidative

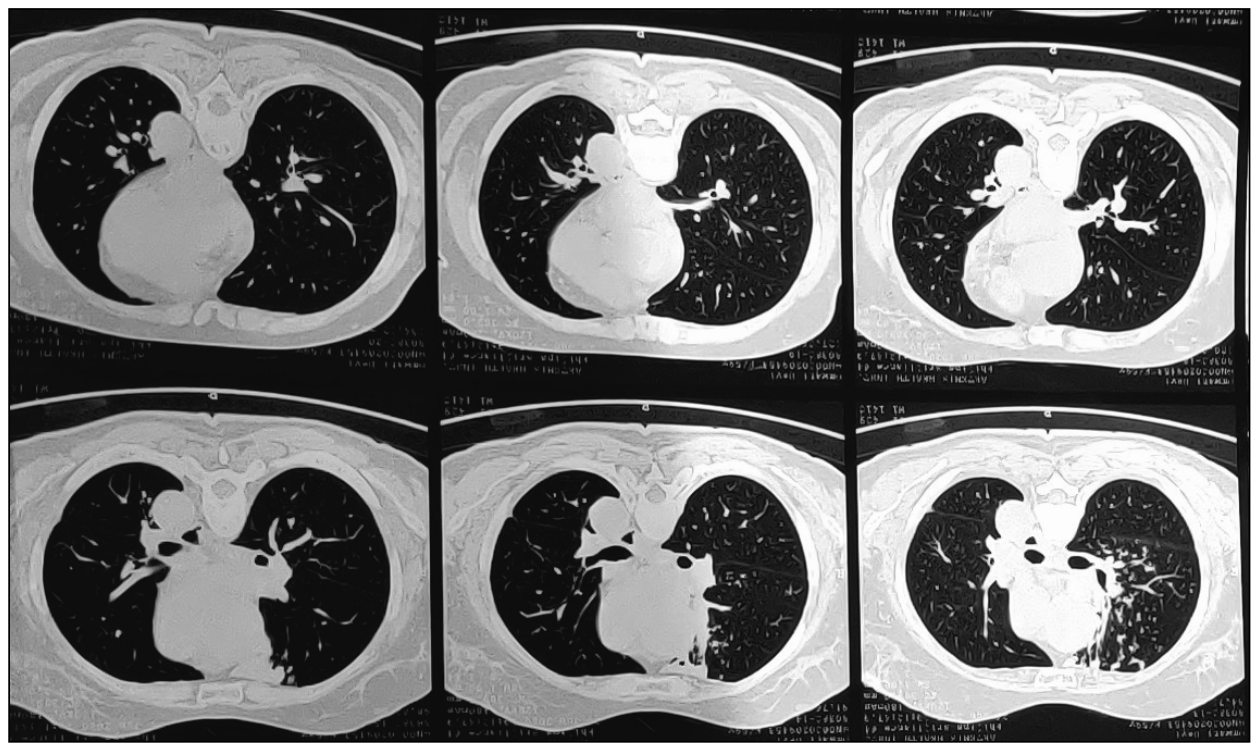

Figure 2. Thorax CT scan revealed multiple ill-defined lesions with variable size scattered throughout both lungs 

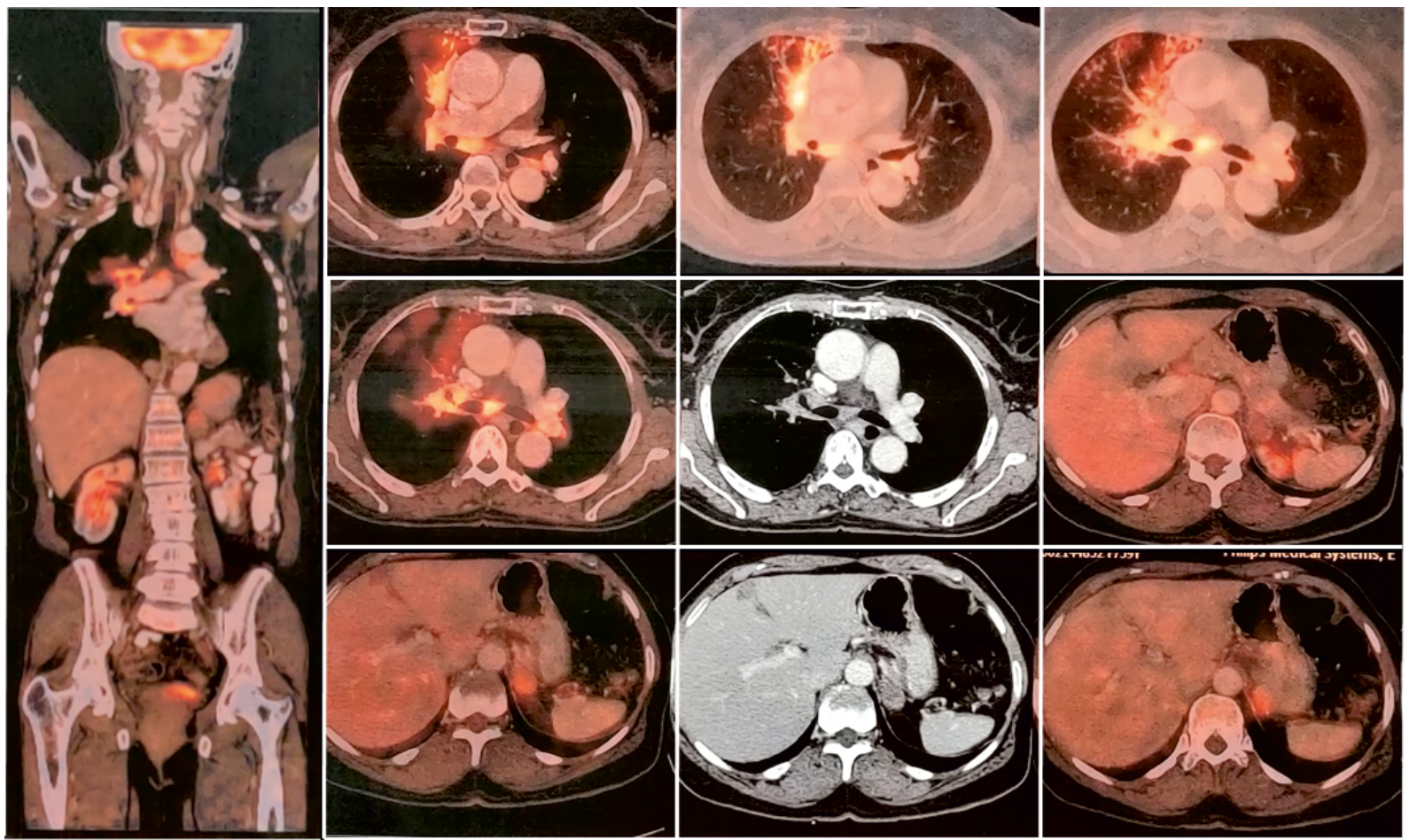

Figure 3. FDG-PET showed high uptake of ${ }^{18} \mathrm{~F}-\mathrm{FDG}$ by local tissues at different foci. The yellow bright spots represented high uptake of ${ }^{18} \mathrm{~F}-\mathrm{FDG}$ by local tissues

lesion with large areas of the internal breakdown was noted in the anterior segments of the bilateral upper lobes. Multiple nodules predominantly sub centimetre size were noted in apical, anterior and posterior segments of the right upper lobe. A few nodules among these were giving rise to the tree-in-bud appearance, representing features of endobronchial spread. A few calcified granulomas were also noted in the bilateral lungs. A well-marginated, homogenous density nodular lesion (HU: 20) was also detected in the left adrenal gland measuring $2.2(\mathrm{AP}) \times 1.6(\mathrm{TS}) \times 2.2(\mathrm{CC}) \mathrm{cm}$ in size (Fig. 3). Since the PET findings showed the treein-bud appearance type of lesions along with multiple calcified centrilobular nodules, they were considered to be suggestive of tubercular aetiology. The clinician further advised the patient to undergo three sputum acid-fast bacilli (AFB) test for tuberculosis detection.

\section{Treatment}

The AFB smear test of sputum for $M$. tuberculosis was conducted in the patient for three consecutive days, of which $M$. tuberculosis was detected in the specimen collected over two consecutive days. The patient was immediately put on anti-TB drugs, comprising R-Cinex, Pyzina, Ethambutol, Benadon and Pentocid, for 3 months initially. The condition of the patient significantly improved over time with a response to the prescribed drugs, coughing reduced and a prominent increase in weight (from $48 \mathrm{~kg}$ to $54 \mathrm{~kg}$ within 2 months) along with improvement in overall health was observed.

\section{Differential diagnosis}

Multiple pulmonary nodules in the elderly are more likely to be diagnosed as metastatic cancer instead of tuberculosis. In the case of lesions primarily in the anterior segments of lungs with multiple other small centrilobular nodules scattered in upper lobes giving rise to the tree-in-bud appearance suggestive of endobronchial spread should be differentially diagnosed. The recurring TB and sarcoidosis mainly infect the upper zones of the lungs. Although immuno-compromised patients or the ones with underlying ailments are more prone to TB infection, sometimes healthy people can also be infected. In the present case, clinicians had analysed the disease from all parameters and exclusion of lung cancer was testified before diagnosing the disease as tuberculosis.

\section{Discussion}

Pulmonary TB and lung metastasis have been found to mimic each other with clinical and radiological features [2]. The common symptoms observed in both the diseases are persistent cough, severe weight loss, weakness (fatigue), loss of appetite, chest pain and 
fever. In the present case, the classic symptoms of TB, such as fever and haemoptysis, were not present, which signalled more toward lung cancer. Even the chest radiography and $C T$ scan findings could not differentiate between TB and lung cancer. Also, the first AFB test could not detect the presence of Mycobacterium either due to a smaller number of bacilli in the sputum or because the sputum specimen was not collected early in the morning. Moreover, the abnormalities on radiological outcomes were ill-defined soft tissue lesions as large as $4-5 \mathrm{~mm}$ associated with multiple scattered centrilobular nodular infiltrates in the whole upper lobe, which are signs of lung cancer/TB concomitant with lung cancer/TB [3]. The radiological outcome of PET/CT is crucial to find out non-pulmonary TB or neoplasm-associated TB; hence, in the present report, the suspicion of cancer in the patient was eliminated only after PET diagnosis. Therefore, the best and a cost-effective way to reduce diagnostic error to deal with chameleon $T B$ is the AFB test examining three sputum specimens over three consecutive days, and further differential diagnosis by CT and PET, if the need arises. Furthermore, sputum cytology for malignancy could also be detected. In developing countries, the practice of sputum tests should be done in all patients with long-lasting cough and severe unintended weight loss to diagnose TB as directed in the Revised National Tuberculosis Control Program (RNTCP) guideline (RNTCP, 2005). Since malignancy can be misdiagnosed in X-ray examination as tuberculosis, a suspected mass on chest X-ray should always be examined through CT/PET scan of the whole body.

Some researchers have linked the development of cancer post tuberculosis [4, 5]. Dacosta and Kinare [6] reported the association of lung cancer and tuberculosis in $\sim 13 \%$ of patients. In another similar study carried out by Cicènas and Vencevičius [7], 2.1\% of cases of co-occurrence of lung cancer and tuberculosis have been observed among 2218 patients. Therefore, it seems that tuberculosis induces some changes in the lungs, such as deformation of bronchi and alveoli, which may lead to epithelial dysplasia, resulting in the possibility of lung cancer manifestation [8, 9]. In addition, lung cancer may induce tuberculosis by activating old foci and dissemination of tuberculosis Mycobacterium $[8,9]$. Therefore, patients who have recovered from tuberculosis or are suffering from tuberculosis should undergo clinical examinations and regular follow-ups for any underlying propagation of lung cancer. In the present report, the patient was advised to undergo a PET scan to rule out any underlying malignancy along with tuberculosis.

To conclude, interpreting clinical and radiological outcomes can defy the differentiation of lung cancer from TB. In such a scenario, dynamic thinking should be adopted by clinicians and a thorough examination of the patient, including radiological and pathological tests, should be conducted/advised.

\section{Contribution}

SKY: Concept, data collection, analysis, review of literature, manuscript preparation; RY: Data collection, analysis. Authors also acknowledge Ms Pooja Yadav for editing the manuscript.

\section{Conflicts of interest}

The authors have none to declare.

\section{References}

1. World Health Organization. Global tuberculosis report 2019. Licence: CC BY-NC-SA 3.0 IGO Geneva, 2019.

2. Navid S, Arzhang S, Mirzaei A, et al. Misleading of the diagnosis of Mycobacterium attributed lung diseases to malignancy due to smear, culture and PCR negative results: A lesson from a case report. Indian J Tuberc. 2020; 67(3): 371-373, doi: 10.1016/j.ijtb.2019.09.002, indexed in Pubmed: 32825871

3. Hammen I. Tuberculosis mimicking lung cancer. Respir Med Case Rep. 2015; 16: 45-47, doi: 10.1016/j.rmcr.2015.06.007, indexed in Pubmed: 26744652

4. Aoki K. Excess Incidence of Lung Cancer among Pulmonary Tuberculosis Patients. Jpn J Clin Oncol. 1993; 23: 205-220, doi: 10.1093/oxfordjournals.jjco.a039636.

5. Wu AH, Fontham ET, Reynolds P, et al. Previous lung diseases and risk of lung cancer among lifetime non-smoking women in the United States. Am J Epidemiol. 1995; 141: 1023-1032.

6. Dacosta NA, Kinare SG. Association of lung carcinoma and tuberculosis. J Postgrad Med. 1991; 37(4): 185-189, indexed in Pubmed: 1841965.

7. Cicenas S, Vencevičius V. Lung cancer in patients with tuberculosis. World J Surg Oncol. 2007; 5(1), doi: 10.1186/1477-7819-5-22.

8. Zhingel' IP, Tsimmerinov IE. [Diagnosis of cancer of the lung in patients with tuberculosis and subjects with post-tuberculosis changes of the lungs]. Probl Tuberk. 1989(6): 26-9?, indexed in Pubmed: 2771907.

9. Braude VI. High incidence of bronchogenic cancer in patients with pulmonary tuberculosis. Probl Tuberk. 1984; 4: 55-58. 